\title{
The role of dental professionals in managing HPV infection and oral cancer
}

\begin{abstract}
Introduction: A dramatic increase in the number of cases of head and neck cancers in the U.S. Oropharyngeal cancers (OPCs) have been found to be linked to strains of high-risk human papillomavirus infections. ${ }^{1}$ The purpose of this paper is to review recent updates on the spread of the Human Papilloma Virus (HPV) infection and the increase of oral cancer, as well as the potential roles of dental professionals in the prevention of HPV infection and oral cancer. Also, to help guide dental professionals about their important role in prevention and patient counselling related to HPV infection and oral cancer.

Methods: The literature review was conducted via the examination of 110 scholarly articles, which were published between 2010 and 2019. For these articles, inclusion and exclusion criteria were applied, which resulted in the selection of 41 sources relevant to the issue. Inclusion criteria were articles related to oral cancer prevalence (epidemiology), HPV, the role of dental professionals, risky sexual practices, HPV vaccine, barriers, and recommendations. For exclusion criteria, articles which were completed outside the United States and Canada, in languages other than English, or were only partially available, were avoided. Additionally, technical articles and articles which precede more recent ones on the same topic were eliminated.
\end{abstract}

Conclusion: Dental professionals are encouraged to be part of this prevention effort against HPV infection.

Keywords: HPV vaccine, prevention, dental professionals
Volume 10 Issue 4 - 2019

\author{
Abdulaziz Hakeem,' Frank Alfred \\ Catalanotto ${ }^{2}$ \\ 'Department of Epidemiology, College of Public Health and \\ Health Professions \& College of Medicine, University of Florida, \\ USA \\ ${ }^{2}$ Department of Community Dentistry and Behavioral Science, \\ College of Dentistry, University of Florida, USA
}

\begin{abstract}
Correspondence: Abdulaziz Hakeem, Department of Epidemiology, College of Public Health and Health Professions \& College of Medicine, University of Florida, 1225 Center Dr., Gainesville, FL 326I I, USA, Tel.: + I-352-222-2722, Email AHakee@dental.ufl.edu
\end{abstract}

Received: July 17, 2019 | Published: August 21, 2019
Abbreviations: HPV, human papilloma virus; OPCs, oropharyngeal cancers; HNSCC, head and neck squamous cell carcinomas

\section{Introduction}

A dramatic increase in the number of cases of head and neck cancers in both the U.S. and globally has drawn the attention of the scientific health community. ${ }^{1}$ Oropharyngeal cancers (OPCs) have been found to be linked to strains of high-risk human papillomavirus infections such as HPV $16,18,31, \& 45 .{ }^{1}$ The purpose of this paper is to review recent updates on the spread of the Human Papilloma Virus (HPV) infection and the increase of oral cancer, as well as the potential roles of dental professionals in the prevention of HPV infection and oral cancer.

The purpose of this literature review is to help guide dental professionals about their important role in prevention and patient counselling related to HPV infection and oral cancer. In this review, we explore the dental professional-patient dynamic and the social barriers around communication about HPV infection and vaccination, as well as how to overcome some of these barriers and the benefits of doing so. Moreover, potential risks and benefits of vaccination will be reviewed.

\section{Methods}

This literature review covers a wide range of subjects from HPV infection, oral cancer prevalence, and risk factors, to recent recommendations about dental professionals' role in prevention. The databases through which we searched were PubMed (both PubMed and Dental Journal collection filter), Cinahl, Embase, Cochrane, Web of Science, and Google/Google Scholar. Throughout the research process I focused on these keywords; HPV, Oral Phargenyeal Carcinoma, Oral Cancer, Dental Professionals or Dentists or "Dental Hygienist", Pediatric Adolescent, Parent, Parental Figure, Risk Factors, Oral Sex, Sexual Contact, Sexually Transmitted Diseases/ Infection/Infections, STD/STI , Risk Communication/Counseling, HPV Mode of Transmission, HPV Guidelines Vaccine, HPV Vaccine", Counseling, Counsel, Recommend, Referral, Promotion, Promote, Interprofessional, and Social Pressures.

The literature review was conducted via the examination of 110 scholarly articles, which were published between 2010 and 2019. For these articles, inclusion and exclusion criteria were applied, which resulted in the selection of 42 papers relevant to the issue. Inclusion criteria were articles related to oral cancer prevalence (epidemiology), $\mathrm{HPV}$, the role of dental professionals, risky sexual practices, HPV vaccine, barriers, and recommendations. For exclusion criteria, articles which were completed outside the United States and Canada, in languages other than English, or were only partially available, were avoided. Additionally, technical articles and articles which precede more recent ones on the same topic were eliminated.

\section{Discussion}

\section{Changing prevalence of oral cancer in the U.S.}

In the 1970s, the spread of HPV 16 infection was considered to be the main cause for the rise in head and neck squamous cell carcinomas (HNSCC). ${ }^{2}$ This cancer usually occurred in lingual and palatine tonsils in younger men. ${ }^{3}$ demonstrated that younger age groups are now being diagnosed with this type of cancer compared to those lately affected by oral cancer. In this case, the people who were the most affected were Caucasians who took part in risky sexual behaviors. 
An article by Martin-Hernan et al. ${ }^{4}$ revealed that cancer was not only found in young non-smokers but in young non-drinkers as well, thus proving a clear link to sexual transmission of HPV. Today, cases of HPV are on the rise, especially among young individuals who do not indulge in typical toxic habits, such as tobacco and/or alcohol. D'Souza et al. ${ }^{5}$ found that HPV OPCs are higher among males than females in the U.S. In a group of people between the ages of 18-25, they found that in males, a positive correlation exists between the number of oral sex partners (within a three-month window) and the level of risk the person faces. However, no analogue for females was found. ${ }^{5}$ Conversely, a negative correlation exists for females between the number of lifetime vaginal sex partners and risk of exposure. ${ }^{5}$ They also reported that increased number (more than five) fellatio partners and a increased (more than 25) vaginal intercourse in a lifetime was linked to an increased risk of HPV OPCs. ${ }^{3}$ Furthermore, they found that men are more challenged bio physiologically to clear an oral HPV infection than women. 5

According to the Centers of Disease Control and Prevention (CDC), an epidemic occurs when the level of disease in a community is higher than expected. The group strata for OPC's caused by HPV were as follows: prevalence according to gender and overall race rate $(18,225$ per 100,000 persons), female (3412 per 100,000 persons) and male $(14,814$ per 100,000 persons), where age prevalence is 62 years in women and 61 years in men. ${ }^{6}$ As the data shows oral cancer should be classified as an epidemic.

In a recent epidemiological article by ${ }^{7}$ the authors mentioned that every year, one of the crucial threats to public health worldwide is that $4.5 \%$ of all cases of cancers are linked with HPV. HPV-linked cancer is responsible for $8.6 \%$ of entire female cancer incidents and $0.8 \%$ of all male cancer incidents and for more than half of all female infection-related cancers in 2012. Cervical cancer cases are mostly linked with HPV (83.0\% and 528,000 incidents) after those cancers of the head and neck $(37,500$ incidents), anus $(35,000$ incidents), penis (12,000 incidents), vagina (12,000 incidents) and vulva (8500 incidents) per year.

\section{Link to HPV}

HPV is a small, double-stranded DNA virus that invades the epithelium and cutaneous tissues. Its prevalence in oral cavities in the adult population is $7.3 \%{ }^{6}$ It can spread through high-risk sexual behavior (i.e., oral sex), typically in the younger population, which can lead to OPC development. ${ }^{2} \mathrm{HPV}$ pathophysiology in OPC begins with the cellular viral infection which mutates the p16 gene, deactivates the retinoblastoma gene and upregulates the p16 gene which makes it an indirect marker. For these reasons, HPV-OPC is examined through a biopsy slide that is stained with p16 immunohistochemistry (HPV surrogate marker). P16 stain positive cancer is more responsive to therapy than other non-HPV OPC's. ${ }^{8,9}$

According to ${ }^{10}$ these cancers appear despite the absence of alcohol or tobacco use. Data revealed that $80 \%$ of the oropharyngeal cancers occurred due to HPV spread. ${ }^{11}$ The issue is that HPV infections create a new class of cancer patient than those associated with classical risk factors (i.e., smoking and drinking). Among all the HPV-related cancers, HPV16 is the most common type. The involvement of HPV 16 and 18 are $73 \%$ (460,000 cases) and HPV 6, 11, 16, 18, 31, 33, 45, 52 , and 58 are $90 \%$ (570,000 cases). Large differences are present in the fraction of cancers referable to the HPV due to region, age group and gender?
Kreimer et al. ${ }^{12}$ systematically reviewed articles from January 1997 to June 2009 to research the prevalence of HPV infection in the oral cavity, and found the individual studies were highly variable. They detected oral HPV in 4581 cancer-free candidates to record the prevalence of HPV16 and other HPV strains. $1.3 \%$ of healthy candidates who were examined tested positive for oral HPV16. Additionally, 3.5-4.5\% of other healthy candidates who were examined for carcinogenic HPV also tested positive. Minute differences were found across genders, resulting in $4.6 \%$ for men and $4.4 \%$ for women. Of all HPV strains detected in the oral cavity of candidates, $28 \%$ were HPV 16. This type is more common anatomically in anogenital areas and less common in the oral cavity; nevertheless, several individuals exhibited carcinogenic oral HPV infections.

In a recent meta-analysis that reviewed the literature published between 1995 and 2017, ${ }^{13}$ they included 66 papers and they found that throughout these OPC's attributed to HPV infection (all HPV types $=7.7 \%$ and high-risk HPV $16=1.4 \%$ ) as for incidences (all HPV types). The incidence rate was 4.38 cases per 1000 person-months, which exhibits a lower occurrence in the oral cavity compared to genitalia and cervices in healthy candidates. This does not negate the potential effects of the carcinogenesis mechanism of high-risk HPV. The possibility to administer smaller doses of radiation/chemotherapy and other preventive measures in HPV-OPCs is exemplified, which makes therapy less stressful than other non-HPV OPCs. ${ }^{14}$

As for HPV testing, the recorded specificity for HPV rinses and swabs are $(92 \%, 95 \% \mathrm{CI}=82-97 \%)$ and sensitivity $(72 \%, 95 \%$ $\mathrm{CI}=45-89 \%$ ) for OPC associated with HPV, which shows a limitation because of the false negatives. ${ }^{15}$ The sensitivity of p16INK4a immunohistochemistry was $94 \%$ high $(95 \%$-confidence interval (CI) $91-97 \%$ ) and the specificity was moderate in diagnosing OPC associated with HPV. ${ }^{16}$

The lack of screening tools to detect HPV-related cases of cancer (besides cervical cancer) and the ongoing investigation on the safety and effectiveness of HPV vaccines strengthen the utilization of these vaccines to arrest the entire spectrum of illness. In order to anticipate the elevated influence of HPV vaccination on cervical cancer, the perceived increase in incidence rates of head and neck cancers could be interpreted into a large figure of incidences reduced by vaccination of HPV, including the reduced fractions assignable to HPV at these locations. ${ }^{7}$ This led us to the realization that a small fraction of the world population only gives data about high quality cancer incidence. Misunderstanding of the actual burden can happen due to the inadequate data, and also by ignoring and skipping the appeal for retardation process. So, lack of data inhibits the assessment of interventions. For the global number of genital warts cases, there is a need for more research as no system is available for data collection. More research is also required for head and neck and other anogenital cancers, such as oropharyngeal cancers attributable to HPV, as massive differences have been noticed and for many countries available data is very limited. In order to evaluate the effect of protection on invasive cervical cancer and other cancers associated with HPV, more time is required. However, in countries that introduce the HPV vaccine early, we have seen a clear reduction in prevailing infections of HPV, precancerous lesions, and genital warts. ${ }^{7}$

\section{Prevention}

Vaccination: An article by ${ }^{17}$ studied HPV prevalence in OPCs before a vaccine was introduced to the U.S. from 1995-2005. Among 557 
cases of OPCs, they found that $62 \%$ were positive for vaccine types HPV 16 or $18 \%$ and $72 \%$ were positive for HPV. Prevalence of HPV$16 / 18$ was higher in men (66\%) compared to women (53\%), as well as higher in other racial/ethnic groups (68-80\%) compared to nonHispanic Black patients $(31 \%)$. In the US, this indicated that most OPC's might be prevented by vaccines, but their impact may differ depending on demographic factors. Although the HPV vaccine is effective and safe, it is, however, expensive; nonetheless it is often covered by insurance. Thus, from this imbalance in cost-effectiveness, an unreadiness to discuss HPV vaccine emerged, specifically in the world of dental professionals. ${ }^{18}$

By taking the HPV vaccine before becoming sexually involved, at- risk communities can decrease their likelihood of contracting HPV OPC's. Additionally, current recommendations have offered a catch-up vaccination for people of both genders under the age of $26 .{ }^{19}$ HPV OPCs can be tested through p16 immunohistochemical staining, which helps to identify different indications aside from those caused by tobacco.

The strong correlation between health and income is apparent for several reasons. First, health is related to productivity of the workforce, as healthy people have more energy to perform, enhanced mental health, and more time spent working. Secondly, the economy correlates with a better education, which is affected by health and well-being. Healthy children are often a good investment for a family, they often take a greater interest in their education and studies, showing that they attend school for a larger number of years and attain higher cognitive function than their less healthy counterparts. Another factor that impacts home finances is the well-being and resilience of the children. The populations people who are retired and those with higher savings rates often lead healthier lives. Better saving and investment practices contribute to physical and mental stability, and technological growth (drivers of economic progression). Finally, changed demographics creates a clear association between health and economic improvement through the demographic dividend. ${ }^{20}$

Vaccinations provide a host of potential benefits, including children who are healthier and more able to receive a better education because of fewer missed days of school. A large spectrum of benefits, lower medical care costs, and less parental work loss can be used as the new model for the economic evaluation of vaccines. The returns that are estimated by researchers were around $12 \%$ in 2005 and the predictive analysis for 2020 was around $18 \%{ }^{20}$

The role of patient counselling: Sarah $\mathrm{L} \mathrm{Goff}^{21}$ in an article aimed to identify and explore the patient-physician discussions about the HPV vaccine and connections between the selected features and vaccine uptake. Qualitative content analysis is used to recognize the communication themes that reveal the chances of refinement in vaccine communication. Patients who got the HPV vaccine were $28 \%$ as demonstrated by the quantitative content analysis, and they were younger on average (17.0 vs. 19.6 years old). There is an increase in the overall vaccine administration for the targeted age groups having lower vaccination rates, with the youngest and oldest patients being vaccinated very rarely. ${ }^{21}$

Melanie Kornideas ${ }^{22}$ explains that the small HPV vaccination coverage among US adolescents is due to the refusal by the parents, and the consequences of this missed chance is foregoing the possible prevention of cancer. After detailing parents' refusal of the HPV vaccine, Kornideas indicates other parents' willingness as well. According to this report, secondary acceptance of the parents was calculated to be $45 \%$ with $24 \%$ considering to vaccinate in the coming 12 months. The healthcare provider is responsible for the secondary acceptance and providing follow-up counselling about HPV vaccination (odds ratio, 2.16; 95\% confidence interval, 1.42 3.28 ). But such counselling was received by only $53 \%$ of the parents. Secondary acceptance is also linked with acquiring a higher quality HPV vaccine recommendation from a provider. This involves more communication and greater satisfaction with the provider as well as with higher vaccination confidence. One of the common reasons for secondary acceptance is that parents generally describe that age of the child is increasing (45\%), they need more knowledge about HPV vaccine (34\%), and they have collected advice from their provider (33\%). Most parents resist the idea of vaccine initially however more two thirds seemed to be open to the idea of vaccination the second time around. After initially rejecting the vaccine, nationwide most parents intend to have their child vaccinated later down the line. This is phenomenon is referred to as "secondary acceptance" which stimulated by providers who spread the notion of HPV vaccination repeatedly and in a high-quality conversations or dialogues. ${ }^{22}$

The role of dental professionals in prevention: A recent crosssectional study about the effectiveness of dental professionals' screening in the prevention or early diagnosis of oral cancer was conducted in Nova Scotia, Canada. ${ }^{23}$ This study showed that dental hygienists' knowledge worked in theory but was not effective in practice. ${ }^{23}$ In addition, it shed light on the necessity of demonstrating to dental professionals the importance and value of providing early diagnoses of oral cancers and reducing mortality rates.

The public's perception with regards to the spread of HPV might be that this issue falls to physicians, and the connection to dental professionals can be overlooked. Dental professionals have access to oral and pharyngeal examinations any time a patient comes into their clinic. If the roles of dental professionals could be properly activated, they could act as the first line of defense against the spread of HPV, increasing health awareness and assuring safer sexual practices. The role of dental professionals is to fight against HPV OPC as proposed by Thomas Arnell. Dental providers can play an important part in boosting HPV vaccination rates and arresting the cancer. The author evaluated knowledge and current HPV vaccine counselling practices among Vermont dentists and dental hygienists to recognize possible targets for state-level interventions to enhance vaccine coverage. Under the medical care of the parents, the active role of the providers was found to be $90 \%$ and yet only $50 \%$ assume that it is their duty to recommend the HPV vaccine. To recommend a vaccine only $50 \%$ of providers feel knowledgeable, $78.6 \%$ hardly discuss HPV vaccination with their age appropriate patients, and $82 \%$ infrequently recommend the vaccine.

Marur et al. ${ }^{8}$ found that from 1973 to 2004 , one of the causes of HNSCC was HPV. Dental professionals must monitor the viral spread and treatments a patient receives, more so in patients who have HPV-associated cancers and who are at high risk for recurrence. ${ }^{24,25}$ Due to the continuous and evolving nature of HPV-associated HNSCC, refined measures are needed in terms of gaining a deeper understanding of the natural progress, history and the linkage with HPV-related HNSCC. ${ }^{8}$

Lectures and toolkits were given to dental professionals concerning $\mathrm{HPV}$, and after one month, surveys were given to those who received this information. From those who had been surveyed, $67.5 \%$ reported 
they had become more prepared after exposure to the material, and 91.6\% said their knowledge of HPV had increased. HPV education proved to be successful at increasing self-reported knowledge in dental professionals. ${ }^{26}$ In the clinical applications regarding HPV-associated cancer and vaccine administration in oral health, students were assessed in two dental schools. The role of oral health professionals and the correlation coefficient regarding knowledge of HPV vaccinations among students was 0.71 , and was 0.85 for vaccine administration, clearly illustrating the deficit in knowledge around HPV vaccinations. ${ }^{27}$ The assessment questionnaire was tested on dental students to evaluate their comprehension regarding OPC-HPV/ the vaccine. This is an area not frequently discussed among dental students; thus, similar study results can enhance dental care quality and knowledge. ${ }^{27}$

When dental professionals began discussing HPV/HPV-related cancer and risky behaviors, it was suggested they start with educating younger white adults (40s-50s) with active sexual histories, specifically oral sex. Seventy-seven percent of graduate pediatric dental programs believed the HPV vaccine should be discussed with patients and parents. The increased age of respondents was correlated with the idea of discussion of sexual health and its link to OPCs being within the scope of practice of pediatric dentistry $(\mathrm{r}=035, \mathrm{P}=0.005)$. However, only $25 \%$ of respondents currently include information about HPV and the vaccine in their curricula, ${ }^{28}$ which leads to a noticeable gap between what they believe and the application of this belief. Developing protocols to address this issue might help the application of already-available knowledge.

Dental hygienists mentioned a variety of modes for information accesses, but the understanding of OPCs varied. In addition, they considered variables such as patients' characteristics, the environment of dental practice, and HPV-related information, which affected how dental hygienists applied primary and secondary prevention to HPV infection spread. In summary, dental hygienists successfully identified HPV- OPCs' importance and contributed to prevention efforts, including oral cancer screenings and promotion of the HPV vaccine. ${ }^{29}$

The American Academy of Pediatrics, after adopting new communication strategies, has provided guidelines to address the queries of patients regarding HPV vaccines. Typical evidence of HPV vaccine awareness lies in brochure guides, survey questionnaires, educational lectures and seminars for target populations or populations of interest. Chan \& Fakhry ${ }^{30}$ stated that direct communication with dental professionals helps cater to the health needs of patients. Within this process, some potential obstacles include a lack of proper skills and knowledge regarding the sensitivity of HPV, and this creates communication gaps, which delays the adoption of preventive measures. ${ }^{31}$

An article by Vázquez-Otero et al. ${ }^{32}$ suggested initiating the role of dental professionals against HPV prevention and aimed to assess dental professionals' knowledge regarding the connection between HPV and oral cancer. There are some deficiencies regarding this subject and addressing this issue is the first step toward a better solution. ${ }^{32}$

Another article by Naleway et al..$^{33}$ assessed dental providers habits concerning adolescent vaccinations. This was completed by investigating participants' knowledge of vaccine barriers for preventive medical care. Over $80 \%$ of respondents correctly identified HPV, while 44\% discussed vaccination with their patients, and $66 \%$ showed a knowledge deficit. Additionally, $62 \%$ of dental professionals who responded showed uncertainty about their patients' decisions regarding recommendations, and $61 \%$ lacked the time to discuss or promote an HPV vaccine. Furthermore, 13\% of the respondents expressed concerns about the safety of vaccines, while $10 \%$ of the respondents questioned the effectiveness of vaccines. $70 \%$ of parents had concerns about safety and $60 \%$ about effectiveness, but $80 \%$ supported that providers discuss preventive medical care with patients, while only $54 \%$ said they should discuss vaccinations specifically. Dental providers faced barriers when recommending vaccines, including additional education and communication tools, but were comfortable with discussing medical care. ${ }^{33}$

In another article by Daley ${ }^{34}$ which assessed the knowledge of dental professionals, an imbalance was found between dentists and dental hygienists regarding HPV cancer discussion, which in the case of dental hygienists, was due to a low knowledge score specifically around questions about the effect of HPV on pregnancy and how HPV causes herpes. This suggests the importance of training regarding basic knowledge around HPV and how to aid in understanding this potential epidemic. ${ }^{34}$

These authors summed up the barriers as follows: ${ }^{35-40}$

For patients:

a. Patients between the ages of 13-17 were vaccinated more than (9-12) in four states.

b. Younger males from racial minorities received less attention regarding HPV vaccination.

c. Patients are sensitive to the topic.

For providers:

a. They influence the HPV vaccination decision.

b. Comfort level to talk about sex and risky behaviours.

c. They perceived parents to be hesitant.

d. They judged that patients were at low risk for infection.

e. They presented the vaccine as "optional."

f. There are mixed results on the providers' roles in HPV vaccination.

g. Providers are less likely to talk about vaccination when it is an acute visit.

h. Providers need a different set of skills to facilitate this role.

i. There is a financial burden (one of the most frequent barriers).

j. There is a negative perception of vaccines which contributed by the lack of participation of providers in this topic with their patients. In this case, it would be the parents as they make the decision for pre-adolescent and adolescent age groups.

Lastly, one of the greatest barriers are the parents in control of their children's health. A study by Lazalde ${ }^{40}$ was conducted to gauge how comfortable parents of children between the ages of 11 and 17 years were with dental professionals offering HPV vaccines. Of those surveyed, $23 \%$ expressed comfort with dentists administering an HPV vaccine to their children, while $61 \%$ of parents wanted their regular providers to vaccinate their children, and $58 \%$ of the same group 
surveyed wanted their regular providers to track the vaccines. The greatest concern for those who were surveyed was a lack of coverage, which was reported by $30 \%$. In addition, parents were shown to be more comfortable with dental professionals promoting the vaccine and less comfortable with the vaccine delivery. ${ }^{41}$

In an article discussed by Daley ${ }^{41} 33$ dentists (100\% female), 48 dental hygienists ( $42 \%$ female), and 13 opinion leaders were surveyed. The mean age of the dentists was 46.9 years with an average of 19.2 years in practice, and the mean age of the dental hygienists was 45.7 years with an average of 20.7 years in practice. Most dentists practiced in the private sector $(68 \%)$, while most dental hygienists worked in the public sector $(61 \%)$.

Micro-level determinants such as provider self-efficacy and patient factors were the foundation of the discussion. Dentists were uncomfortable talking to patients about HPV because of the disease's sexual nature, an opinion reinforced by opinion leaders. Dentists cited that these conversations may be difficult due to a lack of skills despite their knowledge on HPV, while dental hygienists were more receptive to educating patients. The opinion leaders mentioned that dental providers need additional education and skill-building to educate patients about HPV prevention through motivational interviewing techniques, scripting, and role playing. Patient characteristics could influence the decision to discuss HPV prevention; these included the patient's age, reaction, and awareness of HPV. Dental hygienists stated they needed to build rapport with patients prior to talking about HPV, which would make it easier to determine if a patient would receive the information well. Some patients lack knowledge about HPV and HPV prevention, and dentists mentioned that patients do not typically ask questions about HPV. All groups mentioned that most patients are unaware they are screened for oral cancer, and dentists reported that not all practitioners tell their patients about the screening procedure when it is done. Providing materials in waiting rooms or operatories could lead patients to initiate a conversation about HPV.

Meso-level determinants such as physical practice, environment, practice management issues, and interpersonal practice involvement plays an intermediate role in this paradigm. At this level, participants discussed the roles and communication patterns among the dental team and between dental professionals and patients. Some dental hygienists noted that dentists do not believe the HPV topic is important enough to discuss with patients, while other dental hygienists felt they could discuss HPV. A major limitation for dentists was time. Dentists said they believed dental hygienists should discuss HPV prevention with patients because they had more time, which was concurred by opinion leaders. Another barrier to discussion is privacy, as many dental offices are comprised of open operatories. However, some dentists mentioned having separate consultation rooms where they could hold private conversations with patients. Most of the dental hygienists mentioned that the fee-for-service model hinders preventive efforts. In practices focused on production or "power hygiene," dental hygienists said they felt limited in their ability to build rapport and educate patients. Dentists noted that some practices had a more business-model orientation and might have been concerned with offending patients and losing them to other practices. Notably, dentists working in private practices/clinics or general pediatric offices seemed concerned about bringing sensitive topics to patients, whereas dentists practicing in public health clinics described having an easier time discussing sensitive topics with patients. Opinion leaders described it would be harder for dentists in private practices to have an HPV conversation than those in academic or public practice settings.
Macro-level determinants such as interprofessional issues, state policies, professional roles, and professional organizations have a large influence on how dental providers address HPV. At this level, participants from each stakeholder group described the influence of professional organizations. One focus group of dental hygienists mentioned the American Dental Association (ADA), along with the American Dental Hygienists' Association (ADHA) as sources of information that have helped normalize sensitive topic conversations with patients. Some said they would feel confident talking to patients about HPV and recommending the HPV vaccine if the ADA endorsed it. Opinion leaders' descriptions of their relationship with the ADA varied depending on the organization they represented, which ranged from a close relationship to no relationship at all. Not all organizations responded to the ADA's recommendations similarly. Opinion leaders from organizations with closer ties to the ADA described having an easier time supporting its recommendations, while others were more critical of them. The dental hygienists emphasized that seeing patients is an opportunity to engage in preventive health conversations, not just prophylactic appointments. For the dentists, there was more variation in their perceived role. Dental hygienists mentioned how dental policies vary by state, including the type of continuing education required, the dental hygienists' ability to practice in different settings with or without dentist supervision, dental care services that can be offered in other settings (i.e., a pediatrician's office), what they can talk about with minors, and liability. Among dentists and opinion leaders, there was no specific discussion of state policies relating to discussing HPV. Participants discussed the lack of inter-professional relationships between professions, primarily among dentistry, medicine, and public health, as an impediment to integrating HPV prevention into dental care. The solution-focused component of the focus groups centered on improving perceptions of the profession and the need for dental hygienists to be recognized as experts in oral health care. They suggested these relationships should extend to other health care professions and establish relationships with physicians to be able to refer patients for further education or treatment. A few of the opinion leaders discussed how there is some collaboration with paediatricians or other medical professionals when dentists refer patients. Dentists expressed that the medical community needs to recognize when to refer patients to dentistry for their oral health.

Lastly, dental professionals were examined through focus groups and interviews to determine how they can better promote HPV prevention in their practices. Low self-efficacy was highlighted as the primary factor on the micro-level when discussing HPV prevention. Then, certain factors such as place of practice and interprofessional relationships were identified to have impacted their prevention efforts on the meso-level. On the larger scale, they found that patients' interaction with dental professionals was heavily affected by professional organizations on the macro-level. For prevention to be effective, multi-level approaches are needed that take into consideration the perceptions of dental professionals and their roles, dental education and training, and dental settings. On a meso- and macro-level, it was found that determinants can be defiant to change because of certain cultural practices in dentistry. Since the relation between HPV and OPCs is intimate, it demands prevention expansion to separate this association. Working on self-efficacy can be a beginning to this long journey of HPV prevention and hopefully, future eradication. In conclusion, an understanding of the multilevel determinants impacting this issue is required to inform future interventions. Innovative approaches and interdisciplinary efforts should aim to provide the information and skills needed for dental 
providers to address this complex issue. Future research should focus on developing multi-level approaches to facilitate skill-building and address barriers to HPV prevention. ${ }^{42}$

\section{Recommendations}

Based on this review, it is clear that dental professionals should take a stronger and more proactive role in helping prevent HPV infection and increased rates of oral cancer. Our recommendations based on the literatures that have been reviewed up to and including the papers by: ${ }^{35-40}$

1. Increased awareness regarding the issue from accurate sources is important because it can help eliminate any false data and encourage a healthy attitude toward the HPV vaccine.

2. Understanding a variety of factors such as patients' age and clinic layouts, which affect many attributes such as the need for privacy as patients may get uncomfortable sharing personal information in public.

3. A mandatory emphasis on communication skills among dental professionals with patients is a key factor as many dental professionals have reported that they feel uncomfortable talking about risky sexual behaviour with patients.

4. Another important factor is patients' personal beliefs, as a positive view of the HPV vaccine can highly influence the decision to receive the vaccine.

5. Providers need to be comfortable with discussing the HPV vaccine and risky sexual behaviour. Also, they need to deliver appropriate and clear information when it comes to complex communications surrounding the HPV vaccination. Lastly, they need to strongly emphasize cancer prevention and promote awareness and adhere to CDC recommendations because it can alter the future of HPV OPC increased prevalence.

\section{Author contribution}

Dr. Abdulaziz Hakeem did the search for the articles and wrote this current review paper. Dr. Frank Catalanotto performed extensive review, edit and designing this current literature review.

\section{Funding}

This research received no external funding

\section{Acknowledgments}

None.

\section{Conflicts of interest}

The authors declare no conflict of interest.

\section{References}

1. Auluck A, Hislop G, Bajdik C, et al. Trends in oropharyngeal and oral cavity cancer incidence of human papillomavirus (HPV)-related and HPV-unrelated sites in a multicultural population:The British Columbia experience. Cancer. 2010;1 I6(II):2635-2644.

2. Feller L, Wood NH, Khammissa RA, et al. Human papillomavirusmediated carcinogenesis and HPV-associated oral and oropharyngeal squamous cell carcinoma. Part 2: Human papillomavirus associated oral and oropharyngeal squamous cell carcinoma. Head Face Med. 20I0;6:15.
3. Young D, Xiao CC, Murphy B, et al. Increase in head and neck cancer in younger patients due to human papillomavirus (HPV). Oral Oncol. 20I5;5 I (8):727-730.

4. Martin-Hernan F, Sanchez-Hernandez JG, Cano J, et al. Oral cancer, HPV infection and evidence of sexual transmission. Med Oral Patol Oral Cir Bucal. 2013; 18(3):e439-e444.

5. D'Souza G, Wentz A, Kluz N, et al. Sex differences in risk factors and natural history of oral human papillomavirus infection. J Infect Dis. 2016;213(12):1893-1896.

6. Division of Cancer Prevention and Control, C.f.D.C.a.P. HPV-Associated Cancers Rates by Race and Ethnicity.

7. Serrano B, Brotons M, Bosch FX, et al. Epidemiology and burden of HPV-related disease. Best Pract Res Clin Obstet Gynaecol. 20 I8;47:14-26.

8. Marur S, D'Souza G, Westra WH, et al. HPV-associated head and neck cancer: A virus-related cancer epidemic. Lancet Oncol. 20 I0; I (8):78I789 .

9. Geissler C, Tahtali A, Diensthuber M, et al. The role of p 16 expression as a predictive marker in HPV-positive oral SCCHN--a retrospective single-center study. Anticancer Res. 2013;33(3):913-916.

10. Chaturvedi AK, Anderson WF, Lortet-Tieulent J, et al.Worldwide trends in incidence rates for oral cavity and oropharyngeal cancers.J Clin Oncol. 20|3;3|:4550-4559.

11. Joseph AW, D'Souza G. Epidemiology of human papillomavirus-related head and neck cancer. Otolaryngol Clin N Am. 20I2;45(4):739-764.

12. Kreimer ARI, Bhatia RK, Messeguer AL, et al. Oral human papillomavirus in healthy individuals: A systematic review of the literature. Sex Transm Dis. 2010;37:386-391.

13. Tam S, Fu S, Xu L, et al. The epidemiology of oral human papillomavirus infection in healthy populations: A systematic review and meta-analysis. Oral Oncol. 2018;82:91-99.

14. Yete S, D'Souza W, Saranath D. High-risk human papillomavirus in oral cancer: clinical implications. Oncology. 2018;94(3):133-141.

15. Gipson BJ, Robbins HA, Fakhry C, et al. Sensitivity and specificity of oral HPV detection for HPV-positive head and neck cancer. Oral Oncol. 2018;77:52-56.

16. Prigge ES, Arbyn M, von Knebel Doeberitz M, et al. Diagnostic accuracy of pI6INK4a immunohistochemistry in oropharyngeal squamous cell carcinomas: A systematic review and meta $\square$ analysis. Int J Cancer. 2016;140(5): I 186-1 I 98.

17. Steinau M, Saraiya M, Goodman MT, et al. Human papillomavirus prevalence in oropharyngeal cancer before vaccine introduction, United States. Emerg Infect Dis. 2014;20(5):822-828.

18. Chattopadhyay A, Weatherspoon D, Pinto A. Human papillomavirus and oral cancer:A primer for dental public health professionals. Community Dent Health. 2015;32(2): I 17-128.

19. Cox JT, Palefsky JM. Human Papillomavirus Vaccinations; UpToDate: Waltham, MA, USA.

20. Bloom DE. The value of vaccination. Adv Exp Med Biol. 20I I;697:I-8.

21. Goff SL, Mazor KM, Gagne SJ, et al.Vaccine counseling:A content analysis of patient-physician discussions regarding human papilloma virus vaccine. Vaccine. 201 I;29(43):7343-7349.

22. Kornides ML, McRee AL, Gilkey MB. Parents who decline HPV vaccination: who later accepts and why? Acad Pediatr. 2018;18(2S):S37S43.

23. Tax CL, Haslam SK, Brillant M, et al. Oral cancer screening: knowledge is not enough. Int J Dent Hyg. 2017;15(3):179-186. 
24. Maxwell JH, Grandis JR, Ferris RL. HPV-associated head and neck cancer: unique features of epidemiology and clinical management. Annu Rev Med. 2016;67:9I-10I.

25. Gillison ML, Chaturvedi AK,Anderson WF, et al. Epidemiology of human papillomavirus-positive head and neck squamous cell carcinoma. J Clin Oncol. 20I5;33(29):3235-3242.

26. Shukla A, Nyambose J, Vanucci R, et al. Evaluating the effectiveness of human papillomavirus educational intervention among oral health professionals. J Cancer Educ. 2018.

27. Rutkoski H, Fowler B, Mooney R, et al. Pilot test of survey to assess dental and dental hygiene student human papillomavirus-related oropharyngeal cancer knowledge, perceptions, and clinical practices. J Cancer Educ. 2018;33(4):907-9|4.

28. Hosking YP, Cappelli D, Donly K, et al. HPV vaccination and the role of the pediatric dentist: survey of graduate program directors. Pediatr Dent. 2017;39(5):383-389.

29. Thompson EL, Daley EM, Vamos CA, et al. Health literacy approaches to improving communication between dental hygienists and patients for HPV-related oral cancer prevention.J Dent Hyg. 2017;9I (4):37-45.

30. Gooi Z, Chan JY, Fakhry C. The epidemiology of the human papillomavirus related to oropharyngeal head and neck cancer. Laryngoscope. 2016;126(4):894-900.

31. Pytynia KB, Dahlstrom KR, Sturgis EM. Epidemiology of HPV-associated oropharyngeal cancer. Oral Oncol. 20I4;50(5):380-386.

32. Vázquez-Otero C, Vamos CA, Thompson EL, et al. Assessing dentists' human papillomavirus related health literacy for oropharyngeal cancer prevention.J Am Dent Assoc. 2018;149(1):9-17.
33. Naleway AL, Henninger ML, Waiwaiole LA, et al. Dental provider practices and perceptions regarding adolescent vaccination. J Public Health Dent. 2018;78(2):159-164.

34. Daley EM, Thompson EL, Vamos CA, et al. HPV-related knowledge among dentists and dental hygienists.J Cancer Educ. 20I8;33(4):90I-906.

35. McCave EL. Influential factors in HPV vaccination uptake among providers in four states. J Community Health. 2010;35(6):645-652.

36. Gilkey MB, McRee AL. Provider communication about HPV vaccination: A systematic review. Hum Vaccines Immunother. 20 I 6; I2(6): I454-I 468.

37. Walker KK, Jackson RD, Sommariva S, et al. USA dental health providers' role in HPV vaccine communication and HPV-OPC protection: A systematic review. Hum Vaccines Immunother. 2019;15(7-8):1863-I869.

38. Malo TL, Ali KN, Sutton SK, et al.The content and context of physicians' communication with males about human papillomavirus vaccination. Hum Vaccines Immunother. 2016; I2(6): I5 I I-1518.

39. Griner SB, Thompson EL, Vamos CA, et al. Dental opinion leaders' perspectives on barriers and facilitators to HPV-related prevention. Hum Vaccines Immunother. 2019; I5(7-8): I856-I862.

40. Lazalde GE, Gilkey MB, Kornides ML, et al. Parent perceptions of dentists' role in HPV vaccination. Vaccine. 20I 8;36(4):46I-466.

41. Daley EM, Vamos CA, Thompson E, et al. The role of dental providers in preventing HPV-related diseases: a systems perspective. J Dent Educ. 2019;83(2): I6I-I72. 